\section{Níveis séricos de creatinina: \\ hipercreatininemia em segmento da população adulta de Salvador, Brasil*}

\section{Serum creatinine levels: hypercreatinemia in a segment of the adult population of Salvador, Brazil}

\section{Ines Lessa}

Instituto de Saúde Coletiva

Universidade Federal da Bahia

Rua Dr. Barachísio Lisboa, 3 - Parque Lucaia

Horto Florestal, Salvador, Bahia - Brasil 40.295-120

inlessa@ufba.br

\section{Resumo}

A prevalência da doença renal crônica (DRC) vem aumentando, seu impacto social é importante e a creatinina um dos seus marcadores. Objetivo: Estimar a prevalência da hipercreatininemia em segmento da população adulta de Salvador, explorar a sua associação com fatores de risco cardiovascular e delinear os grupos de maior risco para a DRC. Metodologia: Foram entrevistados em domicílio 2.298 adultos a partir dos 20 anos de idade, sendo 1.439 analisados nesse estudo. Foram efetuadas medidas da pressão arterial, antropométricas e realizados exames bioquímicos. Creatinina sérica $=1.3 \mathrm{mg} /$ dl foi considerada anormal para ambos os sexos. A análise foi exploratória, incluindo análise bivariada e regressão logística múltipla. Resultados: foram observados: prevalência de hipercreatininemia de 3,1\%, (5,2\% nos homens e 1,6\% nas mulheres), maiores prevalências em idades a partir dos 60 anos, com $12,9 \%$, IC $95 \%(4,3 ; 20,3)$ em homens e $7,9 \%$, IC $95 \%(3,0 ; 12,8)$ em mulheres. Os OR ajustados foram significantes para homens, para idosos, negros em relação aos pardos, hipertensos e história passada de acidente vascular cerebral. Associações positivas não significantes foram observadas para diabetes e história familiar de DRC. Isoladamente, a hipercreatininemia só ocorreu em homens, 2,6\%. Conclusão: Na atualidade, os inquéritos sobre prevalência de hipercreatininemia na população são desnecessários, embora seja altamente recomendada a triagem para hipercreatininemia nos grupos de alto risco para a DRC na prática médica de rotina.

Palavras-chave: Hipercreatininemia. Doença Renal Crônica., Prevalência. Fatores de Risco.

*Estudo componente do Projeto Monitoramento das Doenças Cardiovasculares e do Diabetes no Brasil (MONIT), subprojeto Prevalência de Fatores de Risco Cardiovascular e do Diabetes em Salvador, financiado pelo Centro Nacional de Epidemiologia (CENEPI)/Ministério da Saúde, Processo no. 1746/98 em parceria com o Instituto de Saúde Coletiva da Universidade Federal da Bahia. 
The prevalence of chronic kidney disease (CKD) has been increasing, its social impact is great and creatinine is one of its markers. Objective: To estimate the prevalence of hypercreatinemia in a segment of the adult population of Salvador in order to investigate its association with cardiovascular risk factors, and to identify the groups at greatest risk of developing CKD. Methods: A home interview was carried out in a sample of 2,298 individuals 20 years of age and over and a segment of 1,439 was tested for elevated serum creatinine. Blood pressure and anthropometric measurements were taken. Serum creatinine levels $=1.3 \mathrm{mg} / \mathrm{dl}$ were considered abnormal for either sex. Exploratory analysis included bivariate analysis and multiple logistic regression. Results: The total prevalence of hypercreatinemia was $3.1 \%$ (5.2\% in men and $1.6 \%$ in women). In patients 60 years of age and older, prevalence was $12.9 \%, 95 \% \mathrm{CI}(4.3 ; 20.3)$ in men and $7.9 \%$, $95 \%$ CI $(3.0 ; 12.8)$ in women. Adjusted OR were found to be significant for the elderly, Afro-Brazilians, hypertensive patients and those with a past history of stroke. Non-significant associations were found for diabetes and family history of CKD. Isolated hypercreatininemia occurred in $2.6 \%$ of men, 95\% CI (1.9; 3.1). Conclusion: At the present time, population surveys of hypercreatininemia are unnecessary, although serum creatinine screening should be strongly recommended within routine medical practice for individuals at higher risk for developing CKD.

Key Words: Hypercreatininemia. Chronic Kidney Disease. Prevalence. Risk Factors.

\section{Introdução}

Atualmente, a doença renal crônica (DRC) é considerada um problema mundial de saúde pública ${ }^{1}$, mesmo sendo baixas as prevalências populacionais descritas para países da União Européia ${ }^{2,3}$ e apenas para os Estados Unidos registram-se prevalências de até $10,8 \%^{1}$. Muitas das prevalências são estimadas indiretamente ${ }^{2,3}$, sendo parte da epidemiologia da DRC procedente de casuísticas compostas por parentes de doentes renais crônicos, considerados grupo de risco ${ }^{4}$. Os estudos de base populacional são escassos, sendo os tradicionais fatores de risco para as doenças cardiovasculares os predominantes para a $\mathrm{DRC}^{1,5-7}$, com recente inclusão da proteína $\mathrm{C}$ reativa de alta sensibilidade (PCRas) entre eles ${ }^{8}$. Inversamente, a DRC vem sendo relatada como importante fator de risco cardiovascular ${ }^{1,6,9,10}$ e, embora os casos leves e moderados sejam referidos como de elevada prevalência, geralmente são assintomáticos, não diagnosticados e não tratados ${ }^{5,11}$.

A creatinina sérica é o marcador mais usado para o rastreamento da "disfunção renal” na população, embora não existam pontos de corte definidos para anormalidade, usando-se muitas vezes o percentil ${ }^{2,12,13}$.

No Brasil, a única informação populacional disponível é o recente estudo de Passos et al, realizado em Bambuí, Minas Gerais $^{14}$. Esses autores referem, com base na creatinina sérica, prevalência inexpressiva da “disfunção renal” na população com idade inferior a 59 anos e de 5,09\% para os idosos do sexo masculino.

O tratamento clínico da DRC é oneroso e a qualidade de vida dos pacientes deteriora-se com a progressão da doença. O conhecimento da epidemiologia da DRC possibilita impedir a sua instalação ou protelar a sua evolução, tendo em vista que os seus determinantes com maiores prevalências populacionais são a hipertensão arterial e o diabetes.

Com este estudo pretende-se estimar a prevalência da hipercreatininemia em segmento da população adulta de Salvador, ex- 
plorar a sua associação com fatores de risco cardiovascular e delinear os grupos de maior risco para doença renal crônica .

\section{Material e Métodos}

Estudo de corte transversal, exploratório, em subgrupo de $62,6 \%$ ( $n=1.439$ ) dos 2.298 participantes com idade igual ou maior do que 20 anos do subprojeto "Prevalência dos Fatores de Risco Cardiovascular e do Diabetes (FRCV/DM) em Salvador, 2000", projeto MONIT $)^{15}$. A amostra original foi por conglomerados, em 3 etapas: setores censitários, domicílios e participantes dentre os elegíveis do domicílio. Em inquérito domiciliar, todos responderam a questionário sobre FRCV/DM, história pessoal e familiar de doença renal e diálise/hemodiálise. Foram efetuadas 6 medidas da pressão arterial em 2 blocos de 3 medidas cada, com intervalo aproximado de 10 minutos entre os blocos e de duas medidas da circunferência da cintura, medida com fita metálica rígida, na linha natural da cintura, correspondente ao ponto mais estreito do tórax, entre a última costela e a cicatriz umbilical. O esfigmomanômetro foi o OMRON HEM 705 CP, eletrônico, com vantagens metodológicas: facilidade de treinamento e padronização dos entrevistadores; eliminação de vieses relacionados à visão, audição e atenção do entrevistador; impossibilidade de opção por dígitos terminais e não interferência na velocidade de inflação/deflação do manguito. Todos os participantes foram encaminhados para a sede do projeto nos bairros, onde foram medidos altura e peso, coletado sangue pós-jejum de $12 \mathrm{~h}$ para dosagens bioquímicas (creatinina sérica, colesterol total, HDLc colesterol, triglicérides, glicemia) e urina para realização do exame de urina . Compareceram 1.546 (67,3\%) entrevistados, com perda real, antes da digitação, de $107(7,0 \%)$ dos que completaram todo o protocolo. Compuseram o subgrupo estudado 1.439 participantes. Todas as medidas e exames laboratoriais foram realizados por métodos e equipes padronizados, conforme recomendações usuais em epidemiologia, $\mathrm{e}$ o LDL calculado pela fórmula LDLc $=$ Colesterol total - [HDLc + (Tg/5)] para valores de triglicerides $<400 \mathrm{mg} / \mathrm{dl}$.

\section{Critérios e definições}

Os FRCV, acrescidos da história familiar da DRC são reconhecidos como fatores de risco para DRC:

- raça auto-definida pela cor da pele, como branca, parda e negra;

- escolaridade estratificada em baixa (analfabetos + escolarizados até a $4^{\text {a }}$ série); média (da $5^{\mathrm{a}}$ série do $1^{\mathrm{o}}$ grau até a $3^{\mathrm{a}}$ série incompleta do $2^{\circ}$ grau) e alta $\left(2^{\circ}\right.$ grau completo + superior incompleto ou completo, ou seja, $\geq 11$ anos completos de escola);

- classe social pelo critério da Associação Brasileira de Pesquisa de Mercado (ABPEME), condensada em três grupos: alta $(\mathrm{A} 1+\mathrm{A} 2+\mathrm{B} 1)$, média $(\mathrm{B} 2+\mathrm{C})$ e baixa (D+E);

- tabagismo - fumante atual, qualquer número de cigarros/dia;

- consumo em excesso de álcool - consumo em finais de semana com embriaguez freqüente e/ou consumo diário com ou sem embriaguez;

- sobrepeso, incluindo obesidade-índice de massa corpórea, IMC (peso/altura ${ }^{2}$ ) $\geq 25 \mathrm{~kg} / \mathrm{m}^{2}$ de superfície corporal;

- circunferência da cintura (CC) $\geq 88 \mathrm{~cm}$ para mulheres e $\geq 102 \mathrm{~cm}$ para os homens, independentemente da presença de obesidade generalizada;

- colesterol total $\geq 240 \mathrm{mg} / \mathrm{dl}$, método enzimático Trinder;

- HDL-colesterol (HDL- ${ }_{\mathrm{c}}$ ) $<40 \mathrm{mg} / \mathrm{dl}$ pelo método Labtest;

- triglicerídeos $\geq 200 \mathrm{mg} / \mathrm{dl}$ pelo método Soloni modificado;

- glicemia $\geq 126 \mathrm{mg} / \mathrm{dl}$ ou menor se na presença do diabetes mellitus (DM) sob tratamento;

- creatinina $\geq 1,3 \mathrm{mg} / \mathrm{dl}$, ambos os sexos, conforme especificado para o método Cinético-enzimático;

- proteinúria pelo método qualitativo, $+\mathrm{a}$ +++ ; 
- hipertensão arterial (HA) pelo critério do VI Joint: pressão arterial sistólica $\geq 140 \mathrm{mmHg}$ e/ou pressão arterial diastólica $\geq 90 \mathrm{mmHg}$ ou menor do que esses valores para hipertensos sob tratamento com pressão arterial controlada ${ }^{16}$. A média das 5 últimas medidas foi usada para análise.

Análise - Estimativa da prevalência da hipercreatininemia e intervalos de confiança (IC) a 95\%. Pelo "STATA statistical software" foram exploradas possíveis associações entre as variáveis descritas como FRCV e hipercreatininemia por análise bivariada, com estimativas do "odds ratio" (OR) bruto. Variáveis com valores de p inferiores a 0,07 foram incluídas na análise pela regressão logística múltipla (OR ajustados), retendo no modelo final as associações com p - valor $<0,05$.

O projeto foi aprovado pelo Comitê de Ética Médica do Conselho Regional de Medicina do Estado da Bahia e todos os participantes assinaram Termo de Consentimento Pós-informado.

\section{Resultados}

Dos 1.439 participantes, 609 eram homens, correspondendo a $58,2 \%$ da amostra masculina original ( $\mathrm{n}=1.047)$, e as $830 \mathrm{mu}-$ lheres representaram $66,7 \%$ da amostra feminina de origem $(n=1.250)$. Esses percentuais foram $12,6 \%$ (homens) e 12,3\% (mulheres) superiores à participação de cada sexo na amostra global do MONIT. Variáveis demográficas e sociais dos participantes foram semelhantes às da amostra de origem (p>0,05).

A prevalência global de hipercreatininemia foi de $3,1 \%$, sendo 3,2 vezes maior para os homens $(5,2 \%$ contra $1,6 \%$ nas mulheres). Na análise descritiva, as prevalências de hipercreatininemia aumentaram com a idade em ambos os sexos, IC 95\% significativos para homens dos 40 aos 69 anos e para mulheres a partir dos 70 anos, sendo de $9,5 \%$, IC $95 \%(5,2 ; 13,8)$ para pessoas a partir dos 60 anos, com $12,9 \%$ IC $(4,3 ; 20,3)$ para homens e 7,9 , IC $(3,0 ; 12,8)$ para mulheres. Para as variáveis sócio-demográficas, as razões de prevalência (RP) foram elevadas, desfavoráveis aos homens, exceto para menor escolaridade $(\mathrm{RP}=0,8)$ (Tabela 1$)$.

Na Tabela 2, também descritiva, a única variável significante para mulheres foi a hipertensão arterial (HA), enquanto para os homens foram significantes: IMC, circunferência da cintura, hipertensão, acidente vascular encefálico, colesterol e LDL elevados e HDL baixo. Das RP entre homens e mulheres, o consumo excessivo de álcool pelas mulheres ficou abaixo da unidade. Para todas as demais variáveis, as RP foram desfavoráveis aos homens. No caso específico do diabetes, a razão das prevalências intrasexo mostraram ser a hipercreatininemia 1,3 vezes maior entre homens diabéticos em relação aos não diabéticos, e para mulheres 1,7 vezes maior do que nas não diabéticas, embora sem significância estatística. Para o IMC houve perda da informação para 28 $(4,6 \%)$ homens, um deles com creatinina elevada, e para 40 mulheres $(4,8 \%)$. Nesses casos, as prevalências máximas e mínimas esperadas para hipercreatininemia, em relação às observadas, ou não seriam alteradas (homens com IMC normal) ou estariam sub ou superestimadas em um valor máximo de $0,2 \%$, caso as perdas fossem incluídas na análise. As perdas observadas em outras variáveis foram mínimas (máximo de 6 em uma análise) e dispersas entre os participantes. $\mathrm{O}$ desconhecimento da história familiar de doença renal foi de $15,5 \%$ dos homens e $13,2 \%$ das mulheres, estando entre eles incluídos 4 casos de hipercreatininemia em cada sexo.

Entre os 45 participantes com creatinina elevada, $5(11,1 \%)$ referiram ter doença renal e um $(2,2 \%)$ estava em tratamento por hemodiálise.

Dos hipertensos, 23,2\% já haviam sido hospitalizados por motivo relacionado à doença, bem como 10,3\% dos diabéticos; 124 participantes nunca haviam medido a pressão arterial, $0,8 \%$ deles $(\mathrm{n}=1)$ com creatinina elevada e $650(45,2 \%)$ nunca haviam medido a glicemia, $2,5 \%$ deles $(n=16)$ com hipercreatininemia. 
Tabela 1 - Prevalência de hipercreatininemia por variáveis sócio-demográficas. Salvador, Brasil, 2003.

Table 1 - Prevalence of hypercreatininemia by sociodemographic variables. Salvador, Brazil, 2003

\begin{tabular}{|c|c|c|c|c|c|}
\hline \multirow[b]{2}{*}{ Variáveis } & \multicolumn{2}{|c|}{ Masculino } & \multicolumn{2}{|c|}{ Feminino } & \multirow[b]{2}{*}{$\mathrm{RP}^{*}$} \\
\hline & No. (\%) & $\begin{array}{l}\text { Prevalência, } \\
\text { \% e IC(95\%) }\end{array}$ & No. (\%) & $\begin{array}{l}\text { Prevalência, } \\
\text { \% e IC(95\%) }\end{array}$ & \\
\hline \multicolumn{6}{|l|}{ Idade } \\
\hline 20 a 29 anos & $167(27,4)$ & $2,4(0,1 ; 4,7)$ & $180(21,7)$ & - & \\
\hline 30 a 39 anos & $141(23,2)$ & $2,8(0,1 ; 5,6)$ & $230(27,7)$ & $0,4(0,0 ; 1,3)$ & 7,0 \\
\hline $40-49$ anos & $155(25,4)$ & $5,2(1,6 ; 8,7)$ & $198(23,9)$ & $1,0(0,0 ; 2,4)$ & 5,2 \\
\hline $50-59$ anos & $81(13,3)$ & $8,6(2,4 ; 14,9)$ & $108(13,0)$ & $1,9(0,0 ; 4,4)$ & 4,5 \\
\hline $60-69$ anos & $45(7,4)$ & $11,1(1,6 ; 20,7)$ & $69(8,3)$ & $4,3(0,0 ; 9,3)$ & 2,6 \\
\hline 70 ou mais anos & $20(3,3)$ & $15,0(0,0 ; 32,1)$ & $45(5,4)$ & $13,3(3,0 ; 23,7)$ & 1,1 \\
\hline idosos $(\geq 60 \text { anos })^{* *}$ & $65(10,7)$ & $12,3(4,3 ; 20,3)$ & $114(13,7)$ & $7,9(2,9 ; 12,9)$ & 1,6 \\
\hline \multicolumn{6}{|l|}{ Cor da pele } \\
\hline Branca & $151(25,0)$ & $3,3(0,4 ; 6,2)$ & $218(26,5)$ & $1,8(0,0 ; 3,6)$ & 1,8 \\
\hline Negra & $171(28,3)$ & $9,4(4,9 ; 13,7)$ & $231(28,1)$ & $2,6(0,5 ; 4,7)$ & 3,6 \\
\hline Parda & $282(46,7)$ & $3,5(1,4 ; 5,7)$ & $373(45,4)$ & $0,8(0,0 ; 1,7)$ & 4,4 \\
\hline \multicolumn{6}{|l|}{ Classe social } \\
\hline Baixa & $325(56,4)$ & $4,0(1,9 ; 6,1)$ & $456(56,0)$ & $2,0(0,7 ; 3,2)$ & 2,0 \\
\hline Média & $194(33,7)$ & $6,(2,8 ; 9,6)$ & $289(35,5)$ & $1,0(0,0 ; 2,2)$ & 6,2 \\
\hline Alta & $57(9,9)$ & $7,0(0,2 ; 13,9)$ & $70(8,6)$ & & \\
\hline \multicolumn{6}{|l|}{ Escolaridade } \\
\hline até $4^{\text {a }}$ serie & $25(4,1)$ & $4,0(0,0 ; 12,3)$ & $77(9,3)$ & $5,2(0,1 ; 10,3)$ & 0,8 \\
\hline até $8^{\mathrm{a}}$ série & $247(40,9)$ & $5,7(2,7 ; 8,5)$ & $327(39,5)$ & $1,2(0,0 ; 2,4)$ & 4,8 \\
\hline $2^{\circ}$ grau incompleto & $307(50,8)$ & $3,3(1,3 ; 5,6)$ & $402(48,6)$ & $1,2(0,2 ; 2,3)$ & 2,8 \\
\hline $\begin{array}{l}2^{\circ} \text { grau completo até } \\
\text { superior completo }\end{array}$ & $25(4,1)$ & $16,0(0,6 ; 31,4)$ & $21(2,5)$ & - & - \\
\hline
\end{tabular}

O OR bruto para a história pessoal de doença renal ( $\mathrm{n}=5)$ foi de 7,88 , com amplo e não significante IC $95 \%(0.86 ; 72,01)$. A associação entre proteinúria $\mathrm{x}$ creatinina elevada também não foi significante, com $\mathrm{OR}=$ 2,4 , IC $(0,30 ; 18,74$. Em razão do pequeno número de casos, as estimativas foram realizadas apenas para o total de participantes.

As análises exploratórias - bivariada e a regressão logística múltipla - foram inicialmente realizadas para o grupo totalizado. As variáveis foram dicotomizadas, fixandose aquela selecionada para parâmetro quando mantidos mais de dois estratos dicotomizados, e aparecem na Tabela 3, excluindo-se as variáveis que apresentaram o $\mathrm{p}$ valor muito elevado na análise bruta (lípidas séricas, IMC e circunferência da cintura). $\mathrm{Na}$ bivariada, os OR foram significativos para sexo masculino, grupo etário a partir dos 50 anos, cor negra em relação à parda, HA, acidente vascular cerebral e insuficiência cardíaca, chamando a atenção o OR não significativo para o diabetes. Mantiveram-se significativas após os ajustamentos pela regressão logística apenas as idades a partir dos 60 anos, a raça negra em relação aos pardos, HA e história de AVE.

Somente para os homens foram realizadas as análises em separado, em razão da baixíssima prevalência da hipercreatininemia em mulheres, com grande amplitude dos IC a 95\%. Embora todas as variáveis apresentassem OR brutos com valores elevados, vários deles significativos, os resultados são apresentados apenas para o modelo final 
Tabela 2 - Prevalência de hipercreatininemia por fatores de risco cardiovascular e história familiar de doença renal. Salvador, Brasil, 2003.

Table 2 - Prevalence of hypercreatininemia by cardiovascular risk factors and family history of kidney disease. Salvador, Brazil, 2003

\begin{tabular}{|c|c|c|c|c|c|}
\hline \multirow[b]{2}{*}{ Variáveis } & \multicolumn{2}{|c|}{ Masculino } & \multicolumn{2}{|c|}{ Feminino } & \multirow[b]{2}{*}{$\mathrm{RP}^{*}$} \\
\hline & No. (\%) & $\begin{array}{l}\text { Prevalência, } \\
\text { \% e IC(95\%) }\end{array}$ & No. (\%) & $\begin{array}{l}\text { Prevalência, } \\
\text { \% e IC(95\%) }\end{array}$ & \\
\hline \multicolumn{6}{|l|}{ IMC } \\
\hline Normal & $374(64,3)$ & $3,7(1,7 ; 5,8)$ & $399(50,5)$ & $1,8(0,5 ; 3,2)$ & 2,1 \\
\hline Anormal & $207(35,6)$ & $7,7(4,1 ; 11,4)$ & $391(49,5)$ & $1,5(0,3 ; 2,8)$ & 5,1 \\
\hline \multicolumn{6}{|l|}{ Circunf. Cintura } \\
\hline Normal & $400(66,3)$ & $4,5(2,6 ; 6,5)$ & $777(94,2)$ & $1,4(0,6 ; 2,2)$ & 3,2 \\
\hline Anormal & $203(33,7)$ & $6,4(3,0 ; 9,8)$ & $48(5,8)$ & $4,2(0,0 ; 10,00$ & 1,5 \\
\hline \multicolumn{6}{|c|}{ Consumo de álcool } \\
\hline Não/ moderado & $552(90,6)$ & $5,3(3,4 ; 7,1)$ & $817(98,4)$ & $1,6(0,7 ; 2,5)$ & 3,3 \\
\hline Excessivo & $57(9,4)$ & $3,5(0,0 ; 8,4)$ & $13(1,6)$ & $7,7(0,0 ; 24,5)$ & 0,5 \\
\hline \multicolumn{6}{|c|}{ Hist. familiar de DRC } \\
\hline Não & $437(85,0)$ & $5,0(3,0 ; 7,1)$ & $567(78,8)$ & $1,6(0,6 ; 2,6)$ & 3,1 \\
\hline Sim & $77(15,0)$ & $5,2(0,1 ; 10,3)$ & $153(21,3)$ & $0,7(0,0 ; 1,9)$ & 7,4 \\
\hline \multicolumn{6}{|l|}{ Diabetes } \\
\hline Não & $577(94,7)$ & $5,0(3,2 ; 6,8)$ & $754(91,0)$ & $1,6(0,7 ; 2,5$ & 3,1 \\
\hline Sim & $32(5,3)$ & $6,3(0,0 ; 15,1)$ & $75(9,0$ & $2,7(0,0 ; 6,4)$ & 2,3 \\
\hline \multicolumn{6}{|l|}{ HA } \\
\hline Não & $444(72,9)$ & $2,7(1,2 ; 4,7)$ & $560(67,8)$ & - & - \\
\hline $\operatorname{Sim}$ & $165(27,1)$ & $11,5(6,6 ; 16,4)$ & $266(32,2)$ & $5,3(2,6 ; 7,9)$ & 2,2 \\
\hline \multicolumn{6}{|l|}{ AVC } \\
\hline Não & $598(98,2)$ & $4,5(2,8 ; 6,2)$ & $817(98,7)$ & $1,6(0,7 ; 2,5)$ & 2,8 \\
\hline Sim & $11(1,8)$ & $36,4(2,5 ; 70,3)$ & $11(1,3)$ & $9,1(0,0 ; 29,3)$ & 4,0 \\
\hline \multicolumn{6}{|c|}{ Insuficiência cardíaca } \\
\hline Não & $599(98,8)$ & $4,8(3,1 ; 6,6)$ & $807(97,8)$ & $1,5(0,6 ; 2,3)$ & 3,2 \\
\hline Sim & $7(1,2)$ & $28,6(0,0 ; 73,7)$ & $18(2,2)$ & $5,6(0,0 ; 17,3)$ & 5,1 \\
\hline \multicolumn{6}{|l|}{ Colesterol } \\
\hline Normal & $445(73,1)$ & $4,7(2,7 ; 6,7)$ & $541(65,3)$ & $1,1(0,2 ; 2,0)$ & 4,3 \\
\hline Anormal & $164(26,9)$ & $6,1(2,4 ; 9,8)$ & $287(34,7)$ & $2,8(0,8 ; 4,7)$ & 2,2 \\
\hline \multicolumn{6}{|l|}{ HDL } \\
\hline Normal & $435(71,5)$ & $5,3(3,2 ; 7,4)$ & $664(80,2)$ & $1,7(0,7 ; 2,6)$ & 3,1 \\
\hline Anormal & $173(28,5)$ & $4,6(1,5 ; 7,8)$ & $164(19,8)$ & $1,8(0,0 ; 3,9)$ & 2,6 \\
\hline \multicolumn{6}{|l|}{ LDL } \\
\hline Normal & $452(74,3)$ & $4,9(2,9 ; 6,9)$ & $537(64,9)$ & $1,3(0,3 ; 2,3)$ & 3,8 \\
\hline Anormal & $156(25,7)$ & $5,8(2,1 ; 9,5)$ & $290(35,1)$ & $2,4(0,6 ; 4,2)$ & 2,4 \\
\hline \multicolumn{6}{|l|}{ Triglicérides } \\
\hline Normal & $513(84,2)$ & $5,3(3,3 ; 7,2)$ & $733(88,4)$ & $1,8(0,8 ; 2,7)$ & 2,9 \\
\hline Anormal & $96(15,8)$ & $4,2(0,1 ; 8,2)$ & $96(11,6)$ & $1,0(0,0 ; 3,1)$ & 4,2 \\
\hline
\end{tabular}

$\mathrm{IC}=$ Intervalo de confiança. $\mathrm{Cl}=$ Confidence Interval

${ }^{*} \mathrm{RP}=$ razão de prevalência, masculino/feminino. ${ }^{*} P R=$ Male/female prevalence ratio;

(Tabela 4), tendo em vista a perda da significância da maioria, incluindo-se entre as que permaneceram significantes a cor da pele negra em relação tanto aos pardos quanto aos brancos, HA e AVC, perdendo a idade a significância estatística. O DM com associação positiva, mas não significante, foi mantido na tabela.

A Tabela 5 resume os dados sobre prevalências de hipercreatininemia para 
Tabela 3 - OR brutas e ajustadas para associações entre variáveis selecionadas e Creatinina sérica elevada em adultos de Salvador, Brasil, 2003.

Table 3 - Overall and adjusted OR for associations between selected variables and high serum Creatinine in adults in Salvador, Brazil, 2003.

\begin{tabular}{|c|c|c|c|c|c|c|}
\hline Variáveis & $\mathrm{P} *(\%)$ & OR bruta & IC 95\% & OR ajustada & IC 95\% & $\mathrm{p}-$ \\
\hline \multicolumn{7}{|l|}{ Sexo } \\
\hline Feminino & 1,2 & & & & & \\
\hline Masculino & 5,2 & 4,42 & $(2,06 ; 9,49)$ & 5,72 & $(2,37 ; 13,85$ & 0,000 \\
\hline \multicolumn{7}{|l|}{ Idade } \\
\hline 20 a 39 anos & 1,1 & & & & & \\
\hline $40-49$ anos & 2,6 & 2,33 & $(0,83 ; 6,50)$ & 1,57 & $(0,53 ; 40,60)$ & 0,416 \\
\hline $50-59$ anos & 4,3 & 4,02 & $(1,39 ; 11,63)$ & 1,65 & $(0,50 ; 5,47)$ & 0,409 \\
\hline 60 anos ou mais & 9,0 & 8,77 & $(3,48 ; 22,14)$ & 3,70 & $(1,23 ; 11,15)$ & 0,020 \\
\hline \multicolumn{7}{|l|}{ Cor da pele -1 } \\
\hline Parda & 1,4 & & & & & \\
\hline Negra & 5,4 & 4,03 & $(1,74 ; 9,31)$ & 4,17 & $(1,71 ; 10,15)$ & 0,002 \\
\hline \multicolumn{7}{|l|}{ Cor da pele -2 } \\
\hline Branca & 2,7 & & & & & \\
\hline Negra & 5,4 & 2,07 & $(0,92 ; 4,65)$ & 1,77 & $(0,74 ; 4,23)$ & 0,199 \\
\hline \multicolumn{7}{|l|}{ Classe social } \\
\hline Alta & 3,6 & & & & & \\
\hline Média & 3,0 & 0,82 & $(0,26 ; 2,56)$ & 1,65 & $(0,31 ; 8,86)$ & 0,556 \\
\hline Baixa & 2,7 & 0,73 & $(0,24 ; 2,20)$ & 1,79 & $(0,30 ; 10,63)$ & 0,520 \\
\hline \multicolumn{7}{|l|}{ Escolaridade } \\
\hline até a $4^{\mathrm{a}}$ série & 4,4 & & & & & \\
\hline até a $8^{\text {a }}$ série & 3,3 & 0,75 & $(0,25 ; 2,28)$ & 1,24 & $(0,32 ; 4,80)$ & 0,756 \\
\hline $2^{\circ} \mathrm{grau}$ incompleto & 1,8 & 0,40 & $(0,12 ; 1,27)$ & 1,15 & $(0,24 ; 5,61)$ & 0,861 \\
\hline$\geq 2^{\circ}$ grau completo & 9,1 & 2,18 & $(0,52 ; 9,14)$ & 8,29 & $(0,82 ; 83,67)$ & 0,073 \\
\hline \multicolumn{7}{|c|}{ IMC } \\
\hline Normal & 2,6 & & & & & \\
\hline Anormal & 3,2 & 1,22 & $(0,63 ; 2,38)$ & 1,22 & $(0,63 ; 2,38)$ & 0,530 \\
\hline \multicolumn{7}{|l|}{ HA } \\
\hline Não & 1,0 & & & & & \\
\hline Sim & 7,3 & 7,65 & $(3,56 ; 16,44)$ & 5,42 & $(2,22 ; 13,22)$ & 0,000 \\
\hline \multicolumn{7}{|l|}{ AVC } \\
\hline Não & 2,5 & & & & & \\
\hline Sim & 29,4 & 16,30 & $(5,41 ; 49,10)$ & 6,00 & $(1,56 ; 23,10)$ & 0,009 \\
\hline \multicolumn{7}{|c|}{ Insuficiência cardíaca } \\
\hline Não & 2,7 & & & & & \\
\hline Sim & 12,5 & 5,21 & $(1,48 ; 18,34)$ & 2,52 & $(0,46 ; 13,85)$ & 0,286 \\
\hline \multicolumn{7}{|l|}{ Diabetes } \\
\hline Não & 2,9 & & & & & \\
\hline Sim & 2,2 & 0,74 & $(0,18 ; 3,14)$ & 0,35 & $(0,08 ; 1,61)$ & 0,179 \\
\hline
\end{tabular}

* prevalência; *prevalence.

hipertensos e/ou diabéticos e na ausência das duas patologias. Notam-se para HA prevalências significantes para ambos os sexos, maiores para os homens, bem como para estes na ausência das duas patologias, Tabela 5.

\section{Discussão}

A semelhança entre as variáveis do subgrupo estudado com as da amostra original sugerem boa validade interna e externa das informações. As perda de algumas 
Tabela 4 - OR brutas, ajustadas e IC a 95\% das variáveis significantemente associadas à creatinina elevada, sexo masculino (modelo final). Salvador, 2003.

Table 4 - Overall, adjusted OR and 95\%Cl of variables significantly associated with high creatinine, for men (final model). Salvador, 2003.

\begin{tabular}{lcccccc}
\hline Variáveis & Prevalência (\%) & OR bruta & (IC 95\%) & OR ajustada & (IC 95\%) & p-valor \\
\hline $\begin{array}{l}\text { Cor da pele - } 1 \\
\text { Parda }\end{array}$ & 3,5 & & & & & \\
$\begin{array}{l}\text { Negra } \\
\text { Cor da pele - } 2\end{array}$ & 9,4 & 2,81 & $(1,24 ; 6,34)$ & 2,67 & $(1,14 ; 6,24)$ & 0,024 \\
$\begin{array}{l}\text { Branca } \\
\text { Negra }\end{array}$ & 3,3 & & & & & \\
AVC & 9,4 & 3,01 & $(1,08 ; 8,44)$ & 2,95 & $(1,01 ; 8,63)$ & 0,048 \\
Não & 4,5 & & & & & 0,015 \\
Sim & 36,4 & 11,98 & $(3,30 ; 42,42)$ & 5,90 & $(1,42 ; 24,51)$ & \\
HA & 2,7 & & & & & 0,012 \\
Não & 11,5 & 4,67 & $(2,21 ; 9,86)$ & 3,03 & $(1,28 ; 7,18)$ & \\
Sim & & & & & & \\
\hline
\end{tabular}

Tabela 5 - Prevalência de creatinina sérica elevada em adultos com e sem diabetes e/ou hipertensão. Salvador, Brasil, 2000.

Table 5 - Prevalence of high serum creatinine in adults with and without diabetes and/or hypertension. Salvador, Brazil,2000

HA e /ou DM $\quad$ masculino $\quad$ feminino

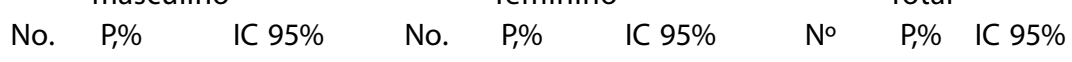

\section{Hipertensos}

- creatinina sérica

\begin{tabular}{|c|c|c|c|c|c|c|c|}
\hline normal & 128 & & & 197 & & & \\
\hline elevad & 18 & 12,3 & $(7,0 ; 17,6)$ & 12 & 5,7 & $(2,6 ; 8,8)$ & \\
\hline
\end{tabular}

Diabéticos

- creatinina sérica

$\begin{array}{lccccc}\text { normal } & 12 & & & 19 & \\ \text { elevada } & 1 & 7,7 & \text { NS } & 0 & 0,0\end{array}$

$\begin{array}{ccc}31 & & \\ 1 & 3,2 & \text { NS }\end{array}$

\section{Diabéticos hipertensos}

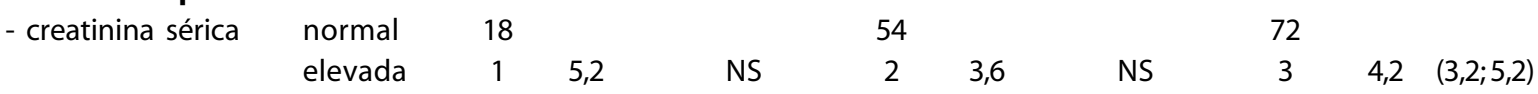

\section{Sem hipertensão ou diabetes}

-creatinina sérica normal

\begin{tabular}{lcccccccc} 
normal & 420 & & \multicolumn{5}{c}{546} & 966 \\
elevada & 11 & 2,6 & $(1,1 ; 4,1)$ & 0 & 0,0 & 11 & 1,1 & NS
\end{tabular}

Total

\begin{tabular}{rlccccccccc} 
com creatinina sérica & normal & 578 & \multicolumn{3}{c}{816} \\
elevada & 31 & 5,1 & $(3,5 ; 6,7)$ & 14 & 1,7 & $(0,82 ; 2,58)$ & 45 & 3,2 & $(2,78 ; 3,9)$ \\
\hline
\end{tabular}

${ }^{*} \mathrm{NS}=$ não significante. ${ }^{*} \mathrm{NS}=$ non significant

medidas do IMC, ou não determinaram alteração na prevalência da hipercreatininemia (homens com IMC normal) ou determinaram sub ou superestimativas máximas de
$0,2 \%$ nos demais participantes, não interferindo nas análises. Para as demais variáveis, as perdas, quando ocorreram, foram inexpressivas. A perda mais significativa foi 
o desconhecimento da história familiar de doença renal, que independe de viés metodológico.

A DRC pode cursar assintomática ${ }^{5,7,11,17}$ e a creatinina sérica elevada é comum ${ }^{5,12}$, mas raramente descrita para segmentos populacionais de não hipertensos, não diabéticos e sem disfunção renal, o que não significa ausência de patologia associada. Em Salvador, 1,1\% da amostra total apresentava hipercreatininemia como evento isolado, com todos os casos detectados no sexo masculino resultando na significativa prevalência de 2,6\% neste sexo. Nenhum homem com hipercreatininemia isolada referiu história pessoal ou familiar de DRC. Nas mesmas condições, prevalência similar de hipercreatininemia está descrita na literatura ${ }^{10}$, possivelmente por causas renais primárias ou secundárias menos prevalentes e assintomáticas, ou por causas externas diversificadas, inclusive uso de fármacos ${ }^{18}$.

A prevalência global da hipercreatininemia é baixa em Salvador, assemelhando-se às mais baixas observadas nos Estados Unidos e na Itália pelo mesmo método $^{2,19}$, e são mais elevadas do que as estimadas por extrapolação a partir de casos de DRC ou insuficiência renal para a população geral de 15 países da União Européia ${ }^{2,3}$, cuja variação referida é de 444 por um milhão da população na Finlândia, e a 773 por milhão da população na Itália² .

A partir dos 60 anos, as prevalências de $12,3 \%$ para homens e $7,9 \%$ para mulheres não são baixas e, além de terem sido mais elevadas do que, respectivamente, os $8,2 \% \mathrm{e}$ $5,1 \%$ descritos para as mesmas idades no Brasil, segundo Passos e cols ${ }^{14}$, confirmam dados da literatura quanto à predominância da hipercreatininemia em idades mais avançadas e no sexo masculino ${ }^{10,12-14}$.

A grande maioria dos participantes identificados como "anormais" tinha hipertensão e/ou diabetes, causas mais prevalentes da DRC na população, sendo a associação entre HA e hipercreatininemia a mais importante desse estudo, em ambos os sexos, 2,2 vezes mais freqüente nos homens pela análise descritiva, e com OR significantes nas análises bruta e ajustada. A associação positiva, mas não significativa, entre diabetes e hipercreatininemia discorda da literatura, porém baixa incidência de DRC foi descrita recentemente em coorte de diabéticos na Itália ${ }^{20}$ e não foi observada no outro estudo brasileiro quando os autores ajustaram pela presença da hipertensão ${ }^{14}$. Além disso, no Brasil - diferente de outros países -, o diabetes ocupa a terceira posição nas terapias de substituição renal, sendo os primeiros lugares ocupados pela glomerulonefrite e pela hipertensão ${ }^{21}$. A associação significativa entre o diabetes e história familiar de $\mathrm{DRC}^{9}$ não observada nesse estudo, mas relatada no Brasil ${ }^{22}$ - baseia-se, não raramente, em casuísticas de familiares de pacientes renais crônicos ${ }^{4}$, vulneráveis ao viés de seleção. Contudo, o desconhecimento da história familiar de nefropatia por aproximadamente $15 \%$ dos participantes pode ter interferido nesse resultado.

A associação do AVC com a hipercreatininemia, mantida após o ajustamento, pode se dever a efeito de colineridade, pela elevada freqüência dehipertensão ( $75 \%$ a $80 \%$ dos casos) em pacientes com AVC em estudos epidemiológicos nacionais ${ }^{23}$. Ademais, esse resultado seria esperado, tendo em vista que, quer sendo causa ou efeito, as associações entre doenças cardiovasculares e doença renal, e destas com as doenças cardiovasculares, estão explícitas na literatura ${ }^{1,7,9,10}$. A insuficiência cardíaca referida como associada à hipercreatininemia ${ }^{12}$ não se confirmou em Salvador.

Por outro lado, mesmo com as dificuldades para a classificação racial no Brasil, o critério usado pelo IBGE, e adotado no estudo, demonstrou diferenças importantes entre negros e pardos quando se considerou o grupo total e entre negros em relação aos pardos e aos brancos para o sexo masculino, mantendo-se a associação desfavorável aos negros. Como nos Estados Unidos ${ }^{11}$, a maior freqüência de nefropatia ocorreu na raça negra, concordando com outros estudos brasileiros mais seletivos ${ }^{24,25}$. Com base nesses dados, não é possível qualquer explicação para a inexistência de gradiente nas 
prevalências da hipercreatininemia nos 3 grupos de cor da pele. Análises futuras, direcionadas para a insuficiência renal, poderão oferecer alguma explicação, pois nessas análises são considerados, simultaneamente, sexo, idade, massa muscular e o valor observado da creatinina sérica, independentemente de ponto de corte (1).

Os resultados sugerem que as abordagens populacionais da hipercreatininemia não são prioritárias para tomada de decisões, e que o mais importante seria a obrigatoriedade do correto cumprimento das normas e estratégias previstas nos programas implementados para controle da hipertensão e do diabetes, mesmo que para estes a associação positiva não tenha sido estatisticamente significante. Isso requer avaliação sistemática, pelo menos anual, da função renal de hipertensos e de diabéticos, registro da creatinina sérica para o monitoramento/vigilância da DRC, com atenção especial para o sexo masculino, idosos e para os negros, que foram considerados os grupos de maior risco, sem exclusão dos demais. O diagnóstico precoce da hipercreatininemia permite estratégias mais agressivas de tratamento das patologias de base e, certamente, resultarão em benefícios pessoais, familiares, sociais e econômicos imensuráveis.

\section{Referências}

1. Sarnak MJ, Levey AS, Schoolwerth AC, Coresh J, Culleton B, Mamm LL et al. Kidney Disease as a risk factor for development of cardiovascular disease. A Statement from the American Heart Association Councils on Kidney in Cardiovascular Disease, High Blood Pressure Research, Clinical, Cardiology, Epidemiology and Prevention. Circulation 2003; 108: 2154-74.

2. Berthoux F. Jones E, Gellert R, Mendel S, Saker L, Briggs D. Epidemiological data of treated end-stage renal failure in the European Union (EU) during the year 1995: report of the European Renal Association Registry and the National Registries. Nephrol Dial Transplant 1999; 14: 2332-42.

3. Magnason RL, Indridason OS, Sigvaldason $\mathrm{H}$, Sigfusson N, Palsson R. Prevalence and progression of CRF in Iceland: a population-based study. Am J Kidney Dis 2002; 40: 955-63.

4. Jurkovitz C, Franch H, Shoham D, Bellenger J, McClellan W. Family members of patients treated for ESRD have high rates of undetected kidney disease. Am J. Kidney Dis 2002; 40: 1173-8.

5. Coresh J, Wei GL, McQuillan G, Brancati FL, Levey AS, Jones $\mathrm{C}$ et al. Prevalence of high blood pressure and elevated serum creatinine level in the United States: findings from the third National Health and Nutrition Examination Survey (1988-1994). Arch Intern Med 2001; 161: 1207-16.

6. Segura J, Campo C, Ruilope LM. How relevant and frequent is the presence of mild renal insufficiency in essential hypertension? J Clin Hypertens (Greenwich) 2002; 4: 332-6.

7. Levin A. Identification of patients and risk factors in chronic kidney disease.- Evaluatting risk factors and therapeutic strategies. Nephrol Dial Transplant. 2001; 16(suppl 7):57-60.
8. Arici M, Walls J. End Stage Renal Disease, Atherosclerosis and Cardiovascular mortality. is C Protein Reactive the missing link? Kidney Int 2001; 59: 407-414.

9. Locatelli F, Pozzoni P, Tentori F, del Vecchio L. Epidemiology of cardiovascular risk in patients with chronic kidney disease. Nephrol Dial Transplant 2003; (S 7): vii2-9.

10. Jones CA, McQuillan GM, Kusek JW, Eberhardt MS, Herman WH, Coresh J et al. Serum creatinine levels in the US population: third National Health and Nutrition Examination Survey. Am J Kidney Dis 1998; 32: 992-9.

11. Crook ED, Washington DO, Flack JM. Screening and prevention of Chronic Kidney disease. J Natl Med Assoc 2002; 94 (S 8): 55S-62S.

12. Fried LF, Shlipak MG, Crump C, Bleyer AJ, Gottdiener JS, Kronmal RA et al. Renal insufficiency as a predictor of cardiovascular outcomes and mortality in elderly individuals. J Am Coll Cardiol 2003; 41: 1364-72.

13. Culleton BF, Larson MG, Evans JC, Wilson PW, Barrett BJ, Parfrey PS et al. Prevalence and correlates of elevated serum creatinine levels: the Framingham Heart Study. Arch Intern Med 1999. 9-23; 159: 1785-90.

14. Passos VM, Barreto SM, Lima-Costa MF.Detection of renal dysfunction based on serum creatinine levels in a Brazilian community: the Bambuí Health and Ageing Study. Braz J Med Biol Res 2003; 36: 393-401.

15. Brasil, Ministério da Saúde/Centro Nacional de Epidemiologia (CENEPI) - Instituto de Saúde Coletiva (ISC). Projeto Monitoramento das Doenças Cardiovasculares e do Diabetes no Brasil (MONIT). Relatório. Ministério da Saúde - CENEPI 2000. 
16. Joint National Committee on Prevention, Detection, Evaluation, and Treatment of High Blood Pressure and National High Blood Pressure Education Program Coordinating Committee. The Sixth report of the Joint National Committee on Prevention, Detection, Evaluation, and Treatment of High Blood Pressure. Archives of Intern Med 1997; 157: 2413-46.

17. Brown WW, Peters RM, Ohmit SE, Keane WF, Collins A, Chen SC et al. Early detection of kidney disease in communnity settings: The Kidney Early Evaluation program (KEEP) 2003; 42: 22-35.

18. Olivares J, Guillen F, Sanchez JJ, Morales-Olivas FJ. Effect of arterial pressure and age on renal function, The "Care for the Kidney" study. Nefrologia 2003; 23: 137-44. (www.pubmed.org, acessado em 27/11/2003).

19. Jha V, Chugh FS. Nephropathy associated with animal, plant and chemichal toxins in the tropics. Semin Nephrol 2003; 23: 49-65 (www.pubmed.org, acessado em 16/11/2003).

20. Bruno G, Boggeri A, Merletti F, Bargero G, Ferrero S, Pagano $\mathrm{G}$ et al. Low incidence of end-stage of renal disease and chronic renal failure in type 2 diabetes: a 10-year prospective study. Diabetes Care 2003; 26: 2353-58.
21. Farias JBL, Bittencourt SS, Alves MAR. Prevalência de nefropatia diabética em adultos com doença renal crônica. Rev Assoc Med Bras 1995; 41: 353-5.

22. Madeira EPQ, Santos PO, Santos SFF, Silva AL, MacIntyre IA, Lopes GS. Familial aggregation of end stage kidney disease in Brazil. Nephron 2002; 91: 666-70.

23. Lessa I. Epidemiologia das doenças cerebrovasculares no Brasil. Rev Soc Cardiol Estado de São Paulo; 1999; 9: 509-18.

24. Lessa I; Brito MM, Fujiko M, Pousada JM. Raça e Nefropatia Diabética em Material de Necrópsia . Arq Bras Med 1996; 70: 565-70.

25. Lopes AA. Hipertensão arterial: fatores Étnicos e Raciais. J Bras Nefrol 1999; 21: 82-4.

recebido em: 20/12/03

versão reformulada apresentada em: 28/04/04

aprovado em: 05/05/04 